\title{
Nonparametric Mobile Speed Estimation in Fading Channels: Performance Analysis and Experimental Results
}

\author{
Hong Zhang, Member, IEEE, and Ali Abdi, Senior Member, IEEE.
}

\begin{abstract}
In this paper we propose a new speed estimation technique, applicable to both mobile and base stations, based on the characteristics in the power spectrum of mobile fading channels. Our analytic performance analysis, verified by Monte Carlo simulations, shows that our low-complexity estimator is not only robust to both Gaussian and non-Gaussian noises, but also insensitive to nonisotropic scattering observed at the mobile. The estimator performs very well in both two- and threedimensional propagation environments. The robustness against both nonisotropic scattering and line of sight can be further increased, by taking advantage of resolvable paths in wideband fading channels, due to the differences among the Doppler spectra observed at different paths. We also extend this technique to base stations with antenna arrays. By exploiting the spatial information, the proposed space-time estimator exhibits excellent performance over a wide range of noise power, nonisotropic scattering, and line-of-sight component, verified by simulation. The utility of the new method is further demonstrated by applying it to the measured data.
\end{abstract}

Index Terms-Doppler estimation, velocity estimation, fading channels, multipath channels, correlation, Doppler spectrum, multiple antennas, macrocell.

\section{INTRODUCTION}

$\mathbf{T}$ HE mobile speed, or the maximum Doppler frequency, reflects the rate of channel fluctuations, and plays a vital role in wireless mobile applications such as handoff, adaptive modulation, equalization, power control, etc. [1] [2]. Therefore, one needs to accurately estimate the mobile speed under different channel conditions. There are three major classes of speed estimation techniques: crossing-based methods, covariance-based methods, and maximum likelihood (ML) based methods. Crossing-based approaches [3] rely on counting the number of received signal's level crossing which is proportional to the mobile speed, while covariancebased algorithms exploit the maximum Doppler frequency information which exists in the sample autocovariance of the received signal [4] [5] [6] [7] [8]. However, both crossingbased and covariance-based speed estimators are sensitive to noise, especially for small Doppler spreads [2] [3] [9]. Although the ML-based estimators [10] [11] are optimal or near optimal, they are complex to implement, need the

This work was presented in part at IEEE Global Telecommunications Conference, St. Louis, MO, 2005.

H. Zhang is with InterDigital Communications Inc., King of Prussia, PA 19406 (Email: hong.zhang@ieee.org)

A. Abdi is with CWCSPR, Department of Electrical and Computer Engineering, New Jersey Institute of Technology, Newark, NJ 07012. (Email: ali.abdi@njit.edu) knowledge of signal-to-noise-ratio (SNR), and require that the noise be Gaussian. Furthermore, the effects of nonisotropic scattering and line-of-sight (LOS) on ML-based estimators are not investigated in [10] and [11]. Wavelet and pattern recognition methods are also used for speed estimation [12] [13], but they are computationally intensive. Recently, two other methods are proposed to estimate Doppler spread by integrating [14] or differentiating [15] [16] the power spectrum density of the mobile fading channel.

In this paper, we propose a new technique, with low complexity, which is not only robust against noise, including both Gaussian and impulsive non-Gaussian, but also insensitive to nonisotropic scattering and LOS. The impact of three dimensional (3-D) propagation which is more likely in urban environments [17] is investigated as well. Two single-antenna-based speed estimators are proposed for the mobile station (MS), relying on the new technique, applicable to both narrowband and wideband channels. Moreover, the performance of the new technique is thoroughly compared with two nonparametric approaches suggested in [14] and [15]. Using the antenna array at the base station (BS), a spacetime estimator is also developed. Mathematical performance analysis of the single-antenna estimators is also presented and confirmed via Monte Carlo simulation.

The organization of this paper is as follows. Signal, channel, and noise models are discussed in Section II, whereas the new speed estimation technique and the associated single-antenna solutions are presented in Section III. Extension to multiple antennas is given in Section IV. Section V includes mathematical performance analysis of single-antenna estimators. Section VI provides numerical results obtained via extensive Monte Carlo simulations. Comparison with measured data is carried out in Section VII, and Section VIII concludes the paper.

\section{Signal, Channel And Noise Models}

The received lowpass complex envelope in a noisy Rician frequency-flat fading channel, in response to an unmodulated carrier, is

$$
z(t)=h(t)+v(t)
$$

where $v(t)$ represents the noise and $h(t)$ includes the random diffuse component $h^{d}(t)$ and the deterministic LOS component

$$
h(t)=\sqrt{\frac{\sigma_{h}^{2}}{K+1}} h^{d}(t)+\sqrt{\frac{K \sigma_{h}^{2}}{K+1}} e^{-j 2 \pi f_{D} t \cos \alpha_{0} \sin \beta_{0}+j \phi_{0}} .
$$


In eq. (2), $h^{d}(t)$ is a zero-mean unit-power complex Gaussian process, $\sigma_{h}^{2}=E\left[|h(t)|^{2}\right]$ is the average power of $h(t)$, and Rice factor $K$ is the ratio of the LOS power to the diffuse power. In the LOS component, $f_{D}=\nu / \lambda=\nu f_{c} / c$ is the maximum Doppler frequency in $\mathrm{Hz}, \nu$ is the MS speed, $\lambda$ is the wavelength, $f_{c}$ is the carrier frequency, and $c$ is the speed of light. We also have $j^{2}=-1, \alpha_{0}$ and $\beta_{0}$ stand for the angle-of-arrival (AOA) in the horizontal and vertical planes, respectively, and $\phi_{0}$ is the phase of the LOS component.

In wireless mobile systems, the complex noise process $v(t)$ in (1) is the superposition of two components: the receiver thermal noise $n_{g}(t)$ and the man-made noise $n_{i m p}(t)$ [18]. The receiver noise is commonly modeled as a white Gaussian process, whereas the man-made noise, generated by electrical equipments such as the vehicle ignition system, neon signs, etc., has an impulsive non-Gaussian characteristic, very different from the Gaussian noise, and appears in the frequency spectrum up to $7 \mathrm{GHz}$ [18]. We use Middleton's Class-A model where the probability density functions (PDF) of the non-Gaussian noise $v(t)$, with zero-mean and variance $\sigma_{v}^{2}$, can be written as [19] [20]

$$
g(v)=e^{-A} \sum_{k=0}^{\infty} \frac{A^{k}}{k ! \pi \sigma_{k}^{2}} \exp \left(-\frac{|v|^{2}}{\sigma_{k}^{2}}\right),
$$

where $\sigma_{k}^{2}=\sigma_{v}^{2}((k / A)+\Gamma) /(1+\Gamma)$, with $\Gamma$ denoting the power ratio of $n_{g}(t)$ over $n_{i m p}(t)$, and parameter $A$ is called the impulsive index. Small values of $A$ indicate a highly impulsive noise whereas large $A$ corresponds to a near-Gaussian one.

The autocorrelation function of $h^{d}(t)$ is defined by $r_{h^{d}}(\tau)=$ $E\left[h^{d}(t) h^{d *}(t+\tau)\right]$ with $*$ as the complex conjugate. In a general 3-D environment with unit-gain isotropic receive antenna , $r_{h^{d}}(\tau)$ can be expressed as [21]

$$
r_{h^{d}}(\tau)=\int_{\theta=0}^{\pi} \int_{\phi=0}^{2 \pi} e^{j 2 \pi f_{D} \tau \cos \phi \sin \theta} q(\theta) p(\phi) \sin \theta d \phi d \theta
$$

in which $p(\phi)$ and $q(\theta) \sin \theta$ are the PDFs of the AOA in the azimuth and elevation planes, respectively. For 2-D propagation, i.e., $q(\theta)=\delta(\theta-\pi / 2)$, where $\delta($.$) is the Dirac$ delta function, together with von Mises PDF for $p(\phi)$, (4) can be written as [22]

$$
r_{h^{d}}(\tau)=\frac{I_{0}\left(\sqrt{\kappa^{2}-4 \pi f_{D}^{2} \tau^{2}+j 4 \pi \kappa f_{D} \tau \cos \alpha}\right)}{I_{0}(\kappa)},
$$

where $\alpha \in[-\pi, \pi)$ is the mean direction of the azimuth AOA, $\kappa \geq 0$ controls the width of the azimuth AOA, and $I_{0}($. is the zero-order modified Bessel function of the first kind. Consequently, the power spectral density (PSD) of $h^{d}(t)$ is given by [22]

$$
\Omega_{h^{d}}(f)=\frac{e^{\frac{\kappa \cos \alpha f}{f_{D}}} \cosh \left(\kappa \sin \alpha \sqrt{1-\left(\frac{f}{f_{D}}\right)^{2}}\right)}{\pi I_{0}(\kappa) \sqrt{f_{D}^{2}-f^{2}}},|f| \leq f_{D},
$$

where $\cosh ($.$) is the hyperbolic cosine.$

For 3-D scattering, we consider the model where the azimuth and elevation AOAs are uniformly distributed over $[-\pi, \pi)$ and $[-\beta, \beta]$, respectively. In this channel, (4) is expressed as [17]

$$
r_{h^{d}}(\tau)=\frac{1}{2 \sin \beta} \int_{\theta=\frac{\pi}{2}-\beta}^{\frac{\pi}{2}+\beta} J_{0}\left(2 \pi f_{D} \tau \sin \theta\right) \sin \theta d \theta,
$$

in which $J_{0}($.$) is the zero-order Bessel function of the first$ kind. The corresponding PSD of $h^{d}(t)$ is [17]

$$
\Omega_{h^{d}}(f)= \begin{cases}\frac{\sin ^{-1}\left(\frac{\sin \beta}{\cos \beta_{f}}\right)}{\pi f_{D} \sin \beta}, & 0 \leq|f|<f_{D} \cos \beta \\ \frac{1}{2 f_{D} \sin \beta}, & f_{D} \cos \beta \leq|f| \leq f_{D}\end{cases}
$$

where $\beta_{f}=\sin ^{-1}\left(f / f_{D}\right)$.

If LOS is present, the autocorrelation function of the Rician fading channel is easy to derive as

$$
r_{h}(\tau)=\frac{\sigma_{h}^{2}}{K+1} r_{h^{d}}(\tau)+\frac{K \sigma_{h}^{2}}{K+1} e^{j 2 \pi f_{D} \tau \cos \alpha_{0} \sin \beta_{0}} .
$$

\section{The New Speed Estimation Algorithm}

By looking at the basic fading spectrum for 2-D isotropic scattering, which is proportional to $\left(f_{D}^{2}-f^{2}\right)^{-1 / 2}$ [2], one can easily observe the two singularities at $f= \pm f_{D}$. These peaks at the maximum Doppler frequency remain to exist, even with nonisotropic scattering, LOS, and 3-D propagation ${ }^{1}$, irrespective of the PDF of noise. Therefore, to estimate $f_{D}$, we propose to estimate the spectrum of the received signal in (1), and then find the frequency at which the spectrum has a maximum. There are many methods to estimate the PSD of a random signal [25]. We have chosen the periodogrambased nonparametric spectrum estimation technique due to its simplicity. We have also observed that more complex nonparametric PSD estimators provide the same performance as the periodogram, when applied to our speed estimation problem. In this section, we propose two $f_{D}$ estimators for frequency-flat and frequency-selective channels, respectively.

\section{A. The Speed Estimator in Narrowband Channels}

Consider the $N$-sample discrete-time version of $z(t)$ with duration $T,\{z[n]=x[n]+j y[n]\}_{n=0}^{N-1}$, with $x$ and $y$ as the real and imaginary parts, respectively, and define the PSD of $\{z[n]\}_{n=0}^{N-1}$ as $S_{z}(\mu)$. In the absence of aliasing, $S_{z}(\mu)$ is the scaled version of the PSD of the continuous signal $z(t), \Omega_{z}(f)$, given by [25]

$$
S_{z}(\mu)=f_{s} \Omega_{z}\left(\mu f_{s}\right),|\mu| \leq 1 / 2,
$$

where $f_{s}=N / T$ is the sampling frequency of $z(t) . S_{z}(\mu)$ can be estimated directly from the data, using the periodogram

\footnotetext{
${ }^{1}$ Peaks at the Doppler spectrum bandlimits are not present for all channel models. See, for example, the one given in [23]. Another example is the Doppler spectra for mobile-to-mobile channels in ad-hoc wireless networks, which often have large peaks near the zero frequency[24]. In such cases, the method of [14] could be a better choice, when the estimator parameters are chosen properly. The channel models used in the present manuscript are commonly used in the literature and are widely adopted by current wireless cellular systems such as GSM and WCDMA.
} 
defined as

$$
\hat{S}_{z}\left(\mu_{k}\right)=\frac{1}{N}\left|\sum_{n=0}^{N-1} z[n] e^{-j 2 \pi \mu_{k} n}\right|^{2}, \mu_{k}=\frac{k}{N},
$$

where $k=1-\frac{N}{2}, \ldots, \frac{N}{2}$ and $N$ is even. Note that (11) can be efficiently computed via fast Fourier transform (FFT). The proposed estimator is then given by

$$
\hat{f}_{D}=f_{s} \times\left|\arg \max _{\mu_{k}} \hat{S}_{z}\left(\mu_{k}\right)\right| .
$$

\section{B. The Speed Estimator in Wideband Channels}

Consider an $I$-tap wideband channel $H(\tau, t)$, with $h_{i}(t)$ being the $i$-th channel gain corresponding to the propagation path with the excess delay $\tau_{i}$

$$
H(\tau, t)=\sum_{i=1}^{I} h_{i}(t) \delta\left(\tau-\tau_{i}\right)
$$

Wideband channel measurements conducted at the MS exhibit a variety of different Doppler spectrum shapes at different propagation paths [26]. As we will see later, this special feature is particularly useful to decrease the estimation error due to some strong nonisotropic scattering and/or LOS which may occur. Based on (12), the speed estimator in frequency selective channels can be written as

$$
\hat{f}_{D}=\max \left\{\hat{f}_{D}^{i}\right\}_{i=1}^{I},{ }^{2}
$$

where $\hat{f}_{D}^{i}=f_{s} \times\left|\arg \max _{\mu_{k}} \hat{S}_{z_{i}}\left(\mu_{k}\right)\right|$. Note that according to (10), $\hat{S}_{z_{i}}\left(\mu_{k}\right)$ is the the estimated Doppler spectrum of the $i$-th path.

\section{The Space-Time Speed Estimation Algorithm}

In this section, we extend our algorithm to a system with multiple receive antennas in narrowband channels. Consider a uniform linear antenna array at the elevated BS of a macrocell, composed of $L$ omnidirectional unit-gain elements, with element spacing $d$. The BS experiences no local scattering, whereas the single antenna MS is surrounded by local scatters. When MS transmits an unmodulated carrier, with the received signals at the $l$-th element $z_{l}(t)=h_{l}(t)+v_{l}(t), l=1,2, \ldots, L$, the space-time crosscorrelation function between $z_{a}(t)$ and $z_{b}(t)$, defined by $r_{z}((b-a) \Delta, \tau)=E\left[z_{a}(t) z_{b}^{*}(t+\tau)\right]$ with $\Delta=d / \lambda$, can be written as

$$
r_{z}((b-a) \Delta, \tau)=r_{h}((b-a) \Delta, \tau)+r_{v}(\tau) \delta_{a-b},
$$

where $r_{v}(\tau)=E\left[v_{l}(t) v_{l}^{*}(t+\tau)\right], l=1,2, \ldots, L$, is the autocorrelation function of the noise component at each branch, $\delta_{c}$ is the Kronecker delta function, 1 for $c=0$ and 0 otherwise,

${ }^{2}$ One way to resolve different propagation paths is to use spreading codes [2]. If different paths cannot be resolved, then one needs to use the proposed narrowband speed estimator. and $r_{h}((b-a) \Delta, \tau)=E\left[h_{a}(t) h_{b}^{*}(t+\tau)\right]$, when considering 2-D scattering, given by [27]

$$
\begin{aligned}
& r_{h}((b-a) \Delta, \tau)=\frac{K \sigma_{h}^{2}}{K+1} \exp \left(j(u+w) \cos \alpha_{0}\right)+\frac{\sigma_{h}^{2}}{K+1} \\
& \times \frac{I_{0}\left(\sqrt{\kappa^{2}-u^{2}-w^{2}-2 u w+j 2 \kappa(u+w) \cos \alpha}\right)}{I_{0}(\kappa)},
\end{aligned}
$$

where $u=2 \pi f_{D} \tau$, and $w=2 \pi(a-b) \Delta$ with $1 \leq a \leq b \leq L$. Note that in (16) we made reasonable assumption of equal receive power $\sigma_{h}^{2}$ and Rician factor $K$ at each antenna. The noise components are independent at different elements, with the same power $\sigma_{v}^{2}$.

According to the experiments conducted at different locations and frequencies [28] [29] [30] [31], the angle spread at the BS is generally small for macrocells in urban, suburban, and rural areas, most often less than $30^{\circ}$, which corresponds to $\kappa \geq 14.6$, and in some cases very small, say, less than $10^{\circ}$, which translates into $\kappa \geq 131.3$ [27] [32]. In such heavily nonisotropic scattering environments, the PDF of the diffuse AOA at the BS can be accurately approximated by a Gaussian PDF with mean $\alpha$ and variance $1 / \kappa$ [27]. Due to the small angle spread at the BS, it is reasonable to assume $\alpha_{0}=\alpha$.

When the channel experiences such heavily nonisotropic scattering, the performance of our estimator degrades. In fact, as proved in Appendix I, the Doppler spectrum $\Omega_{h^{d}}(f)$ of the heavily nonisotropic channel has only one strong peak approximately at $f=f_{D} \cos \alpha$, while the peaks at $f= \pm f_{D}$ virtually disappear. However, for each branch $z_{l}(t)$, still we can use the same technique as (12), which provides us with an accurate estimate of $f_{D} \cos \alpha$

$$
\hat{f}_{D, \alpha}^{l}=f_{s} \times\left|\arg \max _{\mu_{k}} \hat{S}_{z_{l}}\left(\mu_{k}\right)\right| .
$$

On the other hand, by setting $\tau=0$ in (15), together with the Gaussian approximation for AOA, the spatial cross correlation function $r_{z}((b-a) \Delta, 0)=E\left[z_{a}(t) z_{b}^{*}(t)\right], 1 \leq a \leq b \leq L$, can be accurately approximated by [27]

$$
\begin{aligned}
& r_{z}((b-a) \Delta, 0)=r_{h}((b-a) \Delta, 0) \\
& \approx\left\{\frac{\sigma_{h}^{2}}{K+1} \exp \left(-\frac{w^{2} \sin ^{2} \alpha}{2 \kappa}\right)+\frac{K \sigma_{h}^{2}}{K+1}\right\} e^{j w \cos \alpha},
\end{aligned}
$$

where $r_{z}(\cdot, 0)=r_{h}(\cdot, 0), 1 \leq a<b \leq L$, due to the spatial independence of noise components at different branches. Now, we can estimate $\cos \alpha$ via

$$
\widehat{\cos \alpha} \approx \frac{\measuredangle \hat{r}_{z}(\Delta, 0)}{-2 \pi \Delta},
$$

where $\measuredangle$ denotes the phase of a complex number and $\hat{r}_{z}(\Delta, 0)$ is the estimate of $r_{z}(\Delta, 0)=E\left[z_{a}(t) z_{a+1}^{*}(t)\right], \forall a \in[1, L-1]$, given by

$$
\hat{r}_{z}(\Delta, 0)=\frac{1}{L-1} \sum_{l=1}^{L-1} \hat{r}_{z}^{l}(\Delta, 0)
$$

In eq. (20), $\hat{r}_{z}^{l}(\Delta, 0)=N^{-1} \sum_{n=0}^{N-1} z_{l}(n) z_{l+1}^{*}(n), l \in[1, L-$ $1]$, is the $l$-th adjacent-antenna-pair estimate of $r_{z}(\Delta, 0)$.

Finally, $f_{D}$ can be estimated via

$$
\hat{f}_{D}=\hat{f}_{D, \alpha} / \widehat{\cos \alpha}
$$


where $\hat{f}_{D, \alpha}=L^{-1} \sum_{l=1}^{L} \hat{f}_{D, \alpha}^{l}$, and $\hat{f}_{D, \alpha}^{l}$ and $\widehat{\cos \alpha}$ are given in (17) and (19), respectively.

\section{Analytic Performance Assessment}

The performance of the estimator can be measured by using the root mean squared error (RMSE) criterion

$$
\left\{E\left[\left(\hat{f}_{D}-f_{D}\right)^{2}\right]\right\}^{\frac{1}{2}}=\left\{\operatorname{Var}\left[\hat{f}_{D}\right]+\left(E\left[\hat{f}_{D}\right]-f_{D}\right)^{2}\right\}^{\frac{1}{2}},
$$

where the first term in the right-hand side of (22) is the variance and the second stands for the bias of the estimator. In this section, first we derive the key result for the speed estimator in (12) for narrowband channels, and then extend it to estimator in (14) for wideband channels.

It can be shown [33] that for a zero-mean proper complex Gaussian random process $z(t)$, the elements of the sequence $\left\{\hat{S}_{z}\left(\mu_{k}\right)\right\}_{k=1-N / 2}^{N / 2}$ are asymptotically independent as $N \rightarrow$ $\infty$, with an exponential PDF

$$
p_{\hat{S}_{z}\left(\mu_{k}\right)}(s)=\frac{1}{\bar{S}_{z}\left(\mu_{k}\right)} \exp \left\{-\frac{s}{\bar{S}_{z}\left(\mu_{k}\right)}\right\}, s>0,
$$

where $\bar{S}_{z}\left(\mu_{k}\right)$ is the mean of $\hat{S}_{z}\left(\mu_{k}\right)$.

In the absence of both LOS and impulsive noise, $z(t)$ in (1) is a zero-mean proper complex Gaussian random process [34]. Now define $\hat{f}_{\max }=f_{s} \times \arg \max _{\mu_{k}} \hat{S}_{z}\left(\mu_{k}\right)$ so that $\hat{f}_{D}=\left|\hat{f}_{\max }\right|$. Obviously $\hat{f}_{\max }$ is a discrete random variable, taking $N$ values from the set $\left\{f_{s} \mu_{k}\right\}_{k=1-N / 2}^{N / 2}$. Let $\mathscr{E}_{i}$ denote the event where $\mu_{i}$ is the discrete frequency at which $\hat{S}_{z}(\cdot)$ achieves its maximum. Then the probability mass function (PMF) of $\hat{f}_{\max }$ can be obtained as

$$
\begin{aligned}
& p_{\hat{f}_{\max }}\left(f_{i}\right)=\mathrm{P}\left(\hat{f}_{\max }=f_{i}\right)=\mathrm{P}\left(\mathscr{E}_{i}\right) \\
& =\mathrm{P}\left\{\hat{S}_{z}\left(\mu_{i}\right)=\max \left(\left\{\hat{S}_{z}\left(\mu_{k}\right)\right\}_{k=1-N / 2}^{N / 2}\right)\right\} \\
& =\int_{0}^{\infty} \mathrm{P} \underbrace{\left\{\hat{S}_{z}\left(\mu_{1-N / 2}\right)<s, \ldots, \hat{S}_{z}\left(\mu_{N / 2}\right)<s\right\}}_{N-1 \text { terms }} p_{\hat{S}_{z}\left(\mu_{i}\right)}(s) d s,
\end{aligned}
$$

where $f_{i}=f_{s} \times \mu_{i}=i / T, 1-N / 2 \leq i \leq N / 2$. Since $\left\{\hat{S}_{z}\left(\mu_{k}\right)\right\}_{k=1-N / 2}^{N / 2}$ includes independent random variables when $N$ is large, (24) simplifies to

$$
p_{\hat{f}_{\text {max }}}\left(f_{i}\right)=\int_{0}^{\infty} \prod_{l=1-N / 2, l \neq i}^{N / 2} P_{\hat{S}_{z}\left(\mu_{l}\right)}(s) p_{\hat{S}_{z}\left(\mu_{i}\right)}(s) d s,
$$

where $P_{\hat{S}_{z}\left(\mu_{k}\right)}(s)$ is the cumulative distribution function (CDF) of $\hat{S}_{z}\left(\mu_{k}\right)$, given by

$$
P_{\hat{S}_{z}\left(\mu_{k}\right)}(s)=1-\exp \left\{-\frac{s}{\bar{S}_{z}\left(\mu_{k}\right)}\right\}, s>0 .
$$

After substituting (23) and (26) into (25), we obtain

$p_{\hat{f}_{\max }}\left(f_{i}\right)=\int_{0}^{\infty} \prod_{l=1-\frac{N}{2}}^{\frac{N}{2}}\left(1-\exp \left\{-\frac{\bar{S}_{z}\left(\mu_{i}\right)}{\bar{S}_{z}\left(\mu_{l}\right)} s\right\}\right) \frac{e^{-s}}{1-e^{-s}} d s$.
Finally we have

$$
p_{\hat{f}_{D}}\left(f_{i}\right)=p_{\hat{f}_{\text {max }}}\left(f_{i}\right)+p_{\hat{f}_{\text {max }}}\left(f_{1-i}\right), i=0,1, \ldots, \frac{N}{2} .
$$

Note that if $N \rightarrow \infty$, for a fixed $T$, then $\bar{S}_{z}\left(\mu_{i}\right) \rightarrow$ $S_{z}\left(\mu_{i}\right)=f_{s} \Omega_{z}\left(\mu_{i} f_{s}\right)$ [25]. In this case, (27) can be written in terms of the PSD of the continuous-time signal $z(t)$ as

$p_{\hat{f}_{\text {max }}}\left(f_{i}\right)=\int_{0}^{\infty} \prod_{l=1-\frac{N}{2}}^{\frac{N}{2}}\left(1-\exp \left\{-\frac{\Omega_{z}\left(f_{i}\right)}{\Omega_{z}\left(f_{l}\right)} s\right\}\right) \frac{e^{-s}}{1-e^{-s}} d s$.

Based on (29) and (28), it can be shown that with large $N$, as $T$ increases, $p_{\hat{f}_{D}}\left(f_{i}\right)$ becomes more spiky around the true Doppler $f_{D}$ and the estimation error decreases accordingly. Needless to say, the performance of the new estimator can be numerically calculated by substituting (27) and (28) into (22). Monte Carlo simulation results presented in the next section further confirm the accuracy of the analysis of this section.

Now we derive the PDF of the speed estimator in (14) for wideband channels. Since $z_{i}(t)$ 's of different propagation paths can be reasonably modeled as independent processes, the corresponding speed estimates $\hat{f}_{D}^{i}$ in (14) are independent as well. Therefore, similar to the derivation of eq. (25), it can be shown that

$$
p_{\hat{f}_{D}}\left(f_{l}\right)=\sum_{i=1}^{I} \prod_{q=1, q \neq i}^{I} P_{\hat{f}_{D}^{q}}\left(f_{l}\right) p_{\hat{f}_{D}^{i}}\left(f_{l}\right), l=0,1, \ldots, N / 2,
$$

where $P_{\hat{f}_{D}^{q}}\left(f_{l}\right)$ is the CDF of $\hat{f}_{D}^{q}$ and can be readily evaluated using (27) and (28).

\section{Numerical and Monte Carlo Simulation RESULTS}

In this section, we first investigate the performance of the single-antenna speed estimators in (12) and (14), and validate the theoretical performance analysis by Monte Carlo simulations. Then we simulate the performance of the arraybased estimator in (21). The performance comparison with two nonparametric speed estimators proposed in [14] and [15][16] is also conducted in a narrowband channel scenario. All curves are obtained via Monte-Carlo simulation, except those identified by "The" in the legend box, which stands for "Theory".

In each simulation, except mentioned otherwise, 500 independent realizations of $Q$ zero-mean complex Gaussian processes are generated using the spectral method [35], with $N=256$ complex samples per realization, over $T=1$ second, where $Q=1, I, L$, to simulate narrowband and wideband channels for the single-antenna estimators, and the space-time channel for the array-based estimator, respectively. Also we choose the antenna spacing of $\Delta=1 / 2$ for the array-based estimator. If not explicitly mentioned, the noise is Gaussian with a $10 \mathrm{~dB}$ signal-noise-ratio (SNR), defined by $\sigma_{h}^{2} / \sigma_{v}^{2}$, where $\sigma_{v}^{2}$ is the power of the band limited noise with a flat spectrum. The receiver bandwidth $B$ is fixed at $101 \mathrm{~Hz}$, assuming that the largest possible maximum Doppler frequency $f_{D}$ is $101 \mathrm{~Hz}$. 


\section{A. Speed Estimator in a Narrowband Channel}

First we use Monte Carlo simulation to validate our theoretical performance evaluation results in a general scattering environment with 2-D nonisotropic AOA and Gaussian noise in a Rayleigh channel with a single antenna

$$
\begin{aligned}
r_{z}(\tau)= & \sigma_{h}^{2} \frac{I_{0}\left(\sqrt{\kappa^{2}-4 \pi f_{D}^{2} \tau^{2}+j 4 \pi \kappa f_{D} \tau \cos \alpha}\right)}{I_{0}(\kappa)} \\
& +\sigma_{v}^{2} \frac{\sin (2 \pi B \tau)}{2 \pi B \tau} .
\end{aligned}
$$

Then, we examine the effect of LOS, non-Gaussian impulsive noise, and 3-D propagation, on the performance, via Monte Carlo simulation.

We also compare the proposed estimator with two nonparametric estimators. The first one, suggested in [14], uses the normalized cumulative periodogram (NCP) and is given by

$$
\hat{f}_{D}^{B B}=f_{s} \times \frac{p}{N} .
$$

In this estimator. $p$ is the minimum index that satisfies

$$
F_{p}>\psi,
$$

in which

$$
F_{p}=\frac{\hat{S}_{z}\left(\mu_{0}\right)^{\chi}+\sum_{k=1}^{p}\left(\hat{S}_{z}\left(\mu_{k}\right)^{\chi}+\hat{S}_{z}\left(\mu_{-k}\right)^{\chi}\right)}{\sum_{k=1-N / 2}^{N / 2} \hat{S}_{z}\left(\mu_{k}\right)^{\chi}} .
$$

In the simulations, $\chi=1$ and $\psi=0.9$ are chosen, as recommended in [14]. The other nonparametric estimator[15][16] searches for the peak of the differentiated power spectra of fading channels. This estimator can be written as

$$
\hat{f}_{D}^{S M}=f_{s} \times\left|\arg \max _{\mu_{k}} \dot{S}_{z}\left(\mu_{k}\right)\right|,
$$

where

$$
\dot{S}_{z}\left(\mu_{k}\right)=\left|\hat{S}_{z}\left(\mu_{k}\right)-\hat{S}_{z}\left(\mu_{k-1}\right)\right| .
$$

The curves obtained using our proposed estimator is denoted by PSD in the legend boxes.

In Fig. 1, simulations are shown for isotropic scattering, $\kappa=0$, which closely match our theoretical result. The accuracy of the estimator for the $10 \mathrm{~dB}$ SNR is remarkable, when compared with other methods summarized in [3] and [9]. The new estimator also slightly outperforms the nonparametric estimator in (35) because the differentiation in (35) magnifies the effect of noise. For small Dopplers, the proposed estimator shows a better performance than the other nonparametric estimator in (32). This is because the estimator parameters $\chi$ and $\psi$ in estimator (32) depend on the channel conditions which are generally unknown a prior.

The estimation error versus the nonisotropic scattering parameter $\kappa$ with different $\alpha$ 's is shown in Fig. 2, where the true Doppler is $41 \mathrm{~Hz}$. Again, the simulation and theory perfectly match. Note that $0 \leq \kappa \leq 3.3$ represents typical scatterings observed at the mobile, obtained from experimental data collected at a variety of propagation environments [22]. The proposed estimator is generally robust to nonisotropic scattering seen at the mobile and its performance degrades

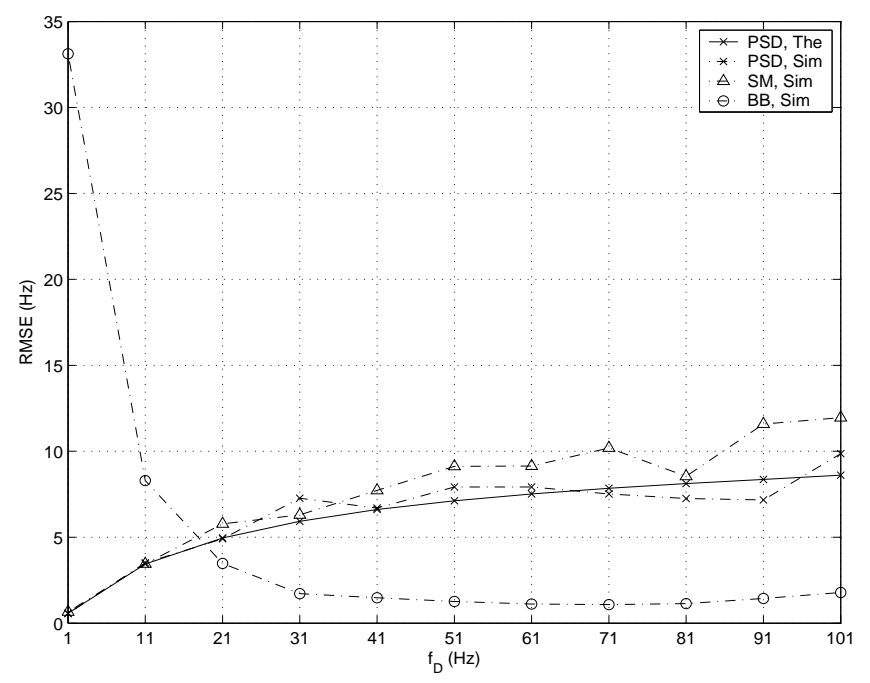

Fig. 1. Performance over a Rayleigh fading channel, isotropic scattering $(\mathrm{SNR}=10 \mathrm{~dB})$.

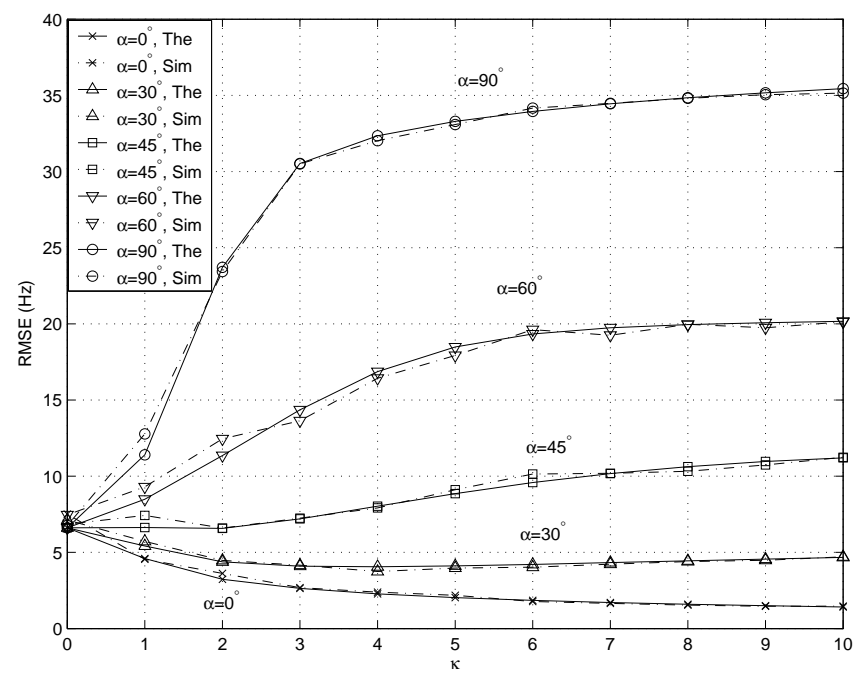

Fig. 2. Effect of $\kappa$ and $\alpha$ in a Rayleigh fading channel $\left(f_{D}=41 \mathrm{~Hz}\right.$, $\mathrm{SNR}=10 \mathrm{~dB}$ )

only for highly directive scenarios with $\alpha \rightarrow 90^{\circ}$. In this case the strongest peak moves away from $\pm f_{D}$. Now we compare the sensitivity to nonisotropic scattering of the three nonparametric estimators. It appears (the simulation results are not shown here due to the space limitation.) that estimator in (32) exhibits better performance at highly directive scenarios with $\alpha \rightarrow 90^{\circ}$.

The estimation error with respect to different SNRs, for $f_{D}=41 \mathrm{~Hz}$, is shown in Fig. 3. As we see, the proposed speed estimator and the one in (35) provide the best performance, even for SNRs as low as $5 \mathrm{~dB}$. On the other hand, the estimator in (32) degrades at low SNRs. This is because the selection of estimator parameters $\chi$ and $\psi$ in (32) depends on the channel characteristics, including the noise level.

Fig. 4 illustrates the estimation error versus $f_{D}$, when the mobile receives a LOS in Rician fading with $K=5$. One observes that the performance degrades to some extent as $\alpha_{0}$ increases because the strongest peak shifts away from $f_{D}$. On 


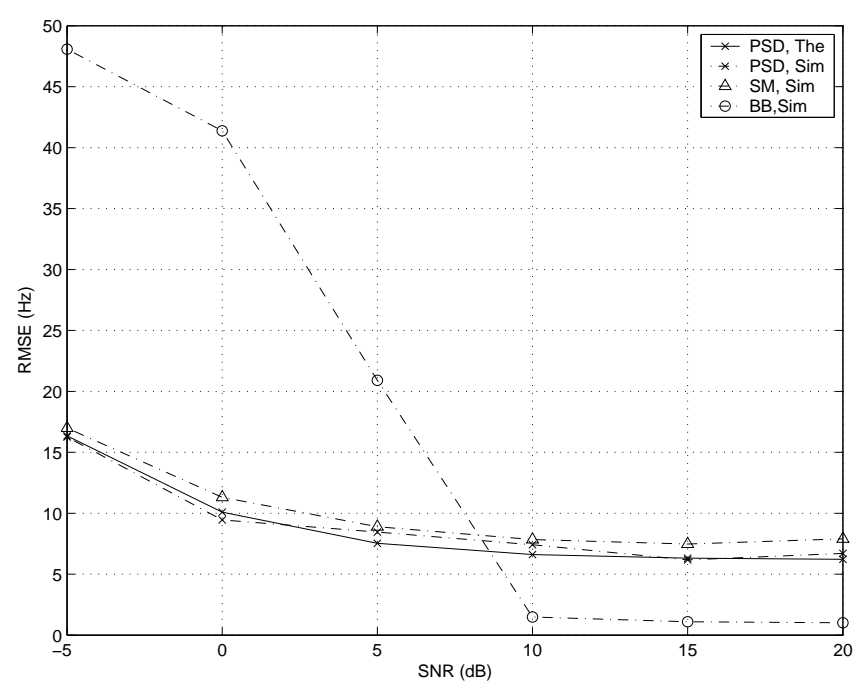

Fig. 3. Effect of SNR in an isotropic Rayleigh fading channel $\left(f_{D}=41\right.$ $\mathrm{Hz})$.

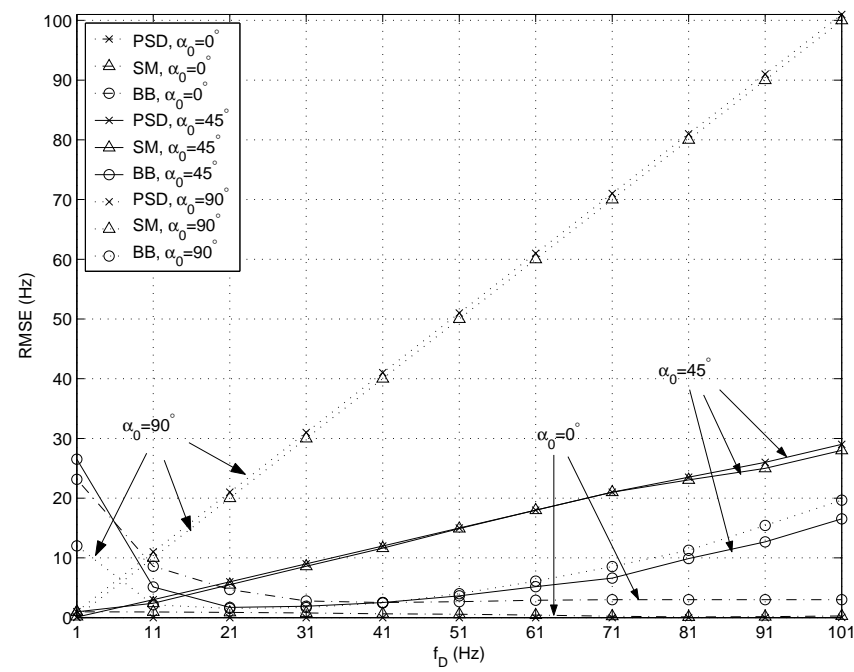

Fig. 4. Performance over a noisy Rician fading channel (SNR=10 dB, $K=5$, $\kappa=2.1, \alpha=10.8^{\circ}$ ).

the other hand, the LOS component is able to improve the estimator's performance, where $\alpha_{0}$ is small. This is due to the impulse generated by LOS at $f=f_{D} \cos \alpha_{0}$. We will see in the next subsection that this dependency on LOS can be easily removed in wideband channels. The comparison in Fig. 4 shows that the new estimator provides almost the same performance as the estimator in (35). It outperforms the estimator in (32) for low Doppler spreads, but degrades at moderate and high Doppler spreads, as $\alpha_{0} \rightarrow 90^{\circ}$.

To look at the effect of the impulsive noise, we consider $(\Gamma=0.01, A=0.1)$ and $(\Gamma=0.01, A=10)$, which indicate that the channel noise is highly impulsive and near-Gaussian, respectively [36]. As shown in Fig. 5, the new estimator is insensitive to the PDF of noise, as expected. The impact of 3-D scattering on speed estimation is shown in Fig. 6. One can see that the new method is still able to provide accurate Doppler estimation over a wide range of mobile speeds. Again, in both cases of $\beta=5^{\circ}$ and $\beta=25^{\circ}$, the proposed estimator

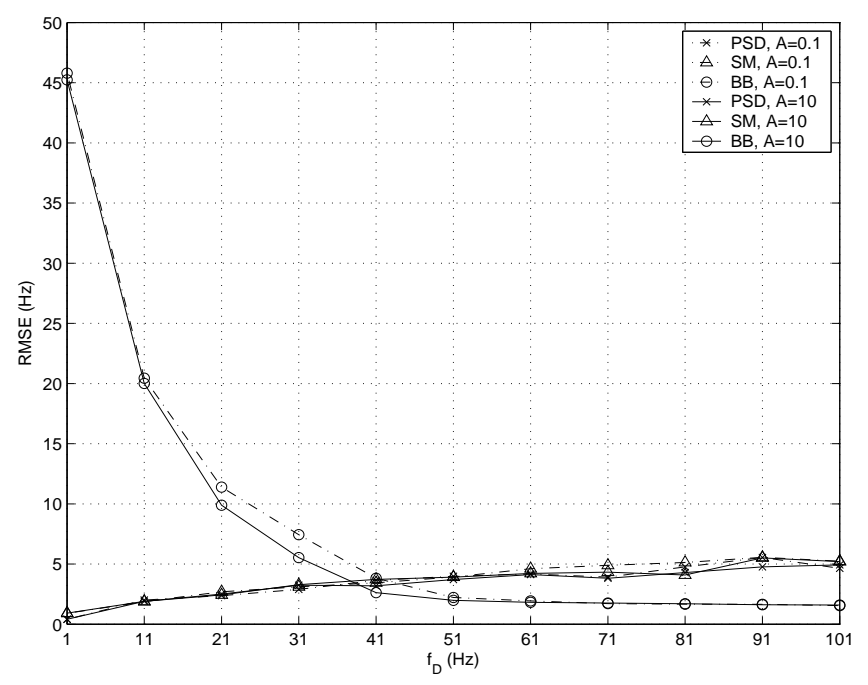

Fig. 5. Performance over a Rayleigh fading channel with impulsive-noise $\left(\kappa=2.1, \alpha=10.8^{\circ}\right)$.

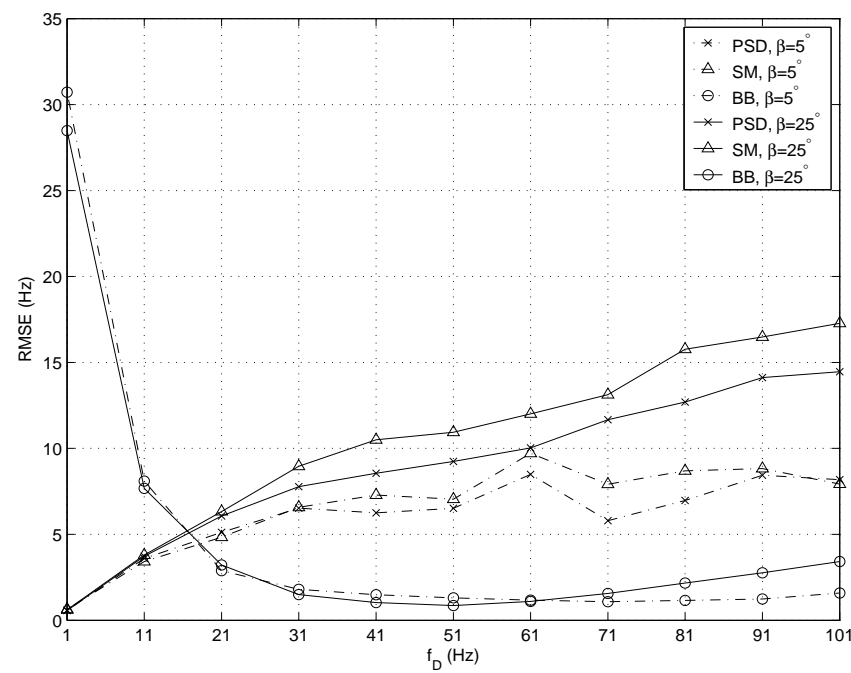

Fig. 6. Performance over a noisy Rayleigh fading channel with threedimensional scattering $(\mathrm{SNR}=10 \mathrm{~dB})$.

demonstrates the same or slightly better performance than the estimator in (35). At small Dopplers, the proposed estimator performs better than the estimator in (32).

Now we discuss the effect of the observation time $T$ and the sample size $N$. First we simulated the estimation error with respect to $T$ in a noiseless isotropic Rayleigh fading channel with $f_{D}=41 \mathrm{~Hz}$ (simulation results not shown due to space limitation.). We observed that, among the three nonparametric estimators, the proposed estimator was more robust to the estimation window length $T$. Even with a small observation time, say, $T=10 \mathrm{~ms}$, the numerical analysis was still accurate and matched the simulation. Moreover, the simulated estimation error with respect to $N$, for a noiseless isotropic Rayleigh fading channel with $f_{D}=41 \mathrm{~Hz}$ and the observation time window $T=0.1 \mathrm{~s}$ showed that all the estimators were fairly insensitive to $N$. We also observed that the theoretical performance analysis was still accurate enough, even with $N$ as small as 32 . 


\section{B. Speed Estimator in a Wideband Channel}

As we have seen in Fig. 2 and Fig. 4, the performance of the proposed narrowband estimator degrades in nonisotropic scattering environments as $\alpha \rightarrow 90^{\circ}$ or in the presence of LOS as $\alpha_{0} \rightarrow 90^{\circ}$. This is due to the spike generated at $f=f_{D} \cos \alpha$ and $f_{D} \cos \alpha_{0}$, respectively. However, according to the experiments conducted at the MS [26], the Doppler spectra observed at different propagation paths of the wideband channel have different shapes. In fact, only the first path or the second path may include LOS or a specular component. Furthermore, the spectra of longer paths are closer to Ushape Clarkes' spectrum, with clear peaks at $\pm f_{D}$. These observations serve as strong motivations for using (14). With $I$ denoting the total number of paths, the total signal power is $\sigma_{h}^{2}=\sum_{i=1}^{I} \sigma_{h_{i}}^{2}$, where $\sigma_{h_{i}}^{2}$ is the received signal power via the $i$ th path. We take the number of available paths as $I=5$ and consider the exponential power delay profile such that $\sigma_{h_{i}}^{2}=\sigma_{h_{1}}^{2} \exp \left\{-\eta\left(\tau_{i}-\tau_{1}\right)\right\}$ [37], where $\tau_{i}=i / 5 \mu \mathrm{s}$, $1 \leq i \leq 5$.

In simulating (14), we only picked up the first three paths as SNR in the last two paths is small. The statistics of the first three paths for scenario 1 are given by $\left(K=5, \alpha_{0}=60^{\circ}, \kappa=3, \alpha=36^{\circ}\right)$, $\left(K=0, \kappa=3, \alpha=36^{\circ}\right)$, and $\left(K=0, \kappa=2.1, \alpha=10.8^{\circ}\right)$, respectively, whereas in scenario 2 we have $\left(K=0, \kappa=10, \alpha=60^{\circ}\right), \quad\left(K=0, \kappa=3, \alpha=36^{\circ}\right), \quad$ and $\left(K=0, \kappa=2.1, \alpha=10.8^{\circ}\right)$. Clearly in scenario 1 there is a LOS in the first path, with mildly nonisotropic scattering in all the paths, whereas in scenario 2 there is no LOS, but strong nonisotropic scattering in the first path and mild ones in the second and third. Although the path SNR decreases by the path delay, one can still obtain sufficiently accurate Doppler estimate, as shown in Fig. 7, due to the robustness of our nonparametric technique to noise, already demonstrated in Fig. 3. Note that the total SNR here is still $10 \mathrm{~dB}$. Obviously by taking advantage of multiple paths, the negative impacts of LOS and strong nonisotropic scattering are avoided. Fig. 8 illustrates the effect of $\eta$ on the performance for scenario 2 , at $f_{D}=41 \mathrm{~Hz}$. When $\eta$ is large, the first path, which is heavily nonisotropic, becomes the dominant path. This is why the performance degrades. In Figs. 7 and 8, our analytic results match the simulations.

\section{The Space-Time Speed Estimator with Multiple Antennas}

The space-time crosscorrelation of fading channel with multiple antennas used in the subsequent simulations is

$$
r_{z}((b-a) \Delta, \tau)=r_{h}((b-a) \Delta, \tau)+\sigma_{v}^{2} \frac{\sin (2 \pi B \tau)}{2 \pi B \tau} \delta_{a-b},
$$

where $r_{h}((b-a) \Delta, \tau)$ is given in (16). As shown in Fig. 2 , heavily nonisotropic scattering, normally experienced by BS in macrocells, could degrade the performance, if the single-antenna estimator in (12) is applied directly. Fig. 9 illustrates the performance of the space-time speed estimator in (21), versus the number of antenna elements $L$, in different propagation scenarios, for $f_{D}=41 \mathrm{~Hz}$. Clearly the estimation error decreases significantly as $L$ increases from one to two,

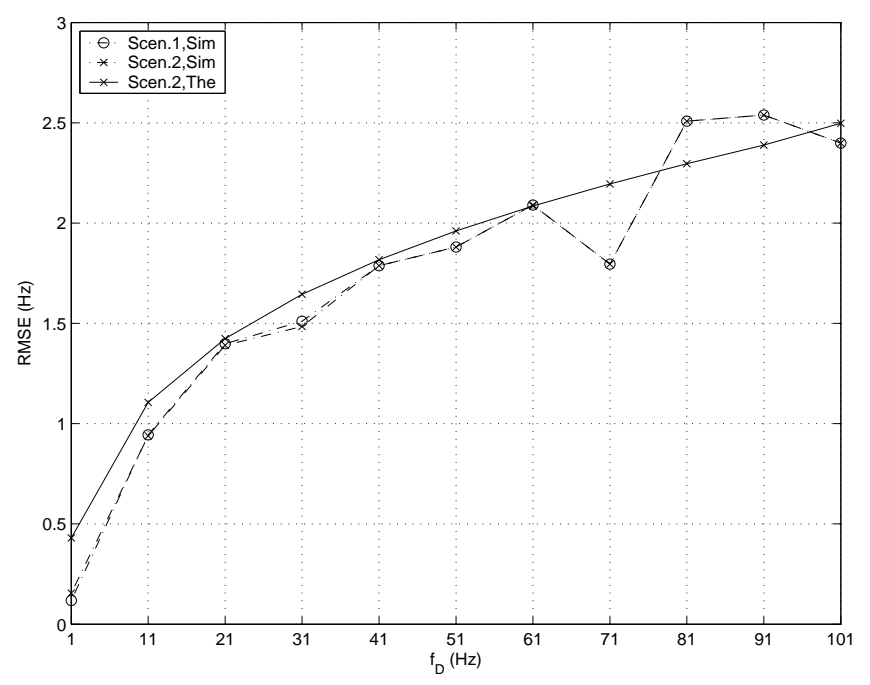

Fig. 7. Performance of the speed estimator in wideband channels $(\eta=1$, $\mathrm{SNR}=10 \mathrm{~dB}$ )

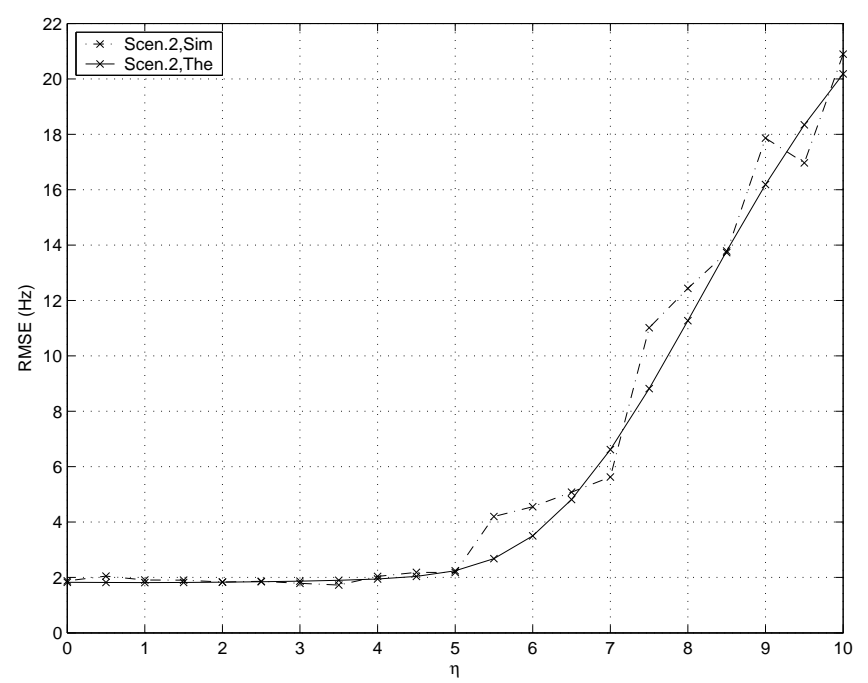

Fig. 8. Effect of $\eta\left(\mathrm{SNR}=10 \mathrm{~dB}, f_{D}=41 \mathrm{~Hz}\right)$

because of the spatial information used. However, further increase in $L$ does not result in much performance gain, as the $L$ estimates $\left\{\hat{f}_{D, \alpha}^{l}\right\}_{l=1}^{L}$ in (21) are highly correlated, due to the small element spacing. This observation indicates that the space-time estimator is actually applicable to BS arrays with any shape or configuration, via using only two adjacent antennas. In the sequel, we choose $L=2$ as well.

Fig. 10 illustrates the RMSE versus $f_{D}$, for both Rayleigh and Rician channels with the scattering scenario $(\kappa=100$, $\left.\alpha=60^{\circ}, \alpha_{0}=60^{\circ}\right)$. Clearly the performance of the arraybased estimator is much better, for different Rice $K$ factors. The effect of $\kappa$ and $\alpha$ on the performance, for $f_{D}=41$ $\mathrm{Hz}$, is shown in Fig. 11. Obviously the array-based estimator provides much smaller estimation error, for different values of $\alpha$. Finally, the effect of element spacing $\Delta$ is simulated in different scattering environments, for $f_{D}=41 \mathrm{~Hz}$. We have also observed that the array-based estimator is fairly insensitive to the choice of $\Delta$. For example, for $f_{D}=41$ 


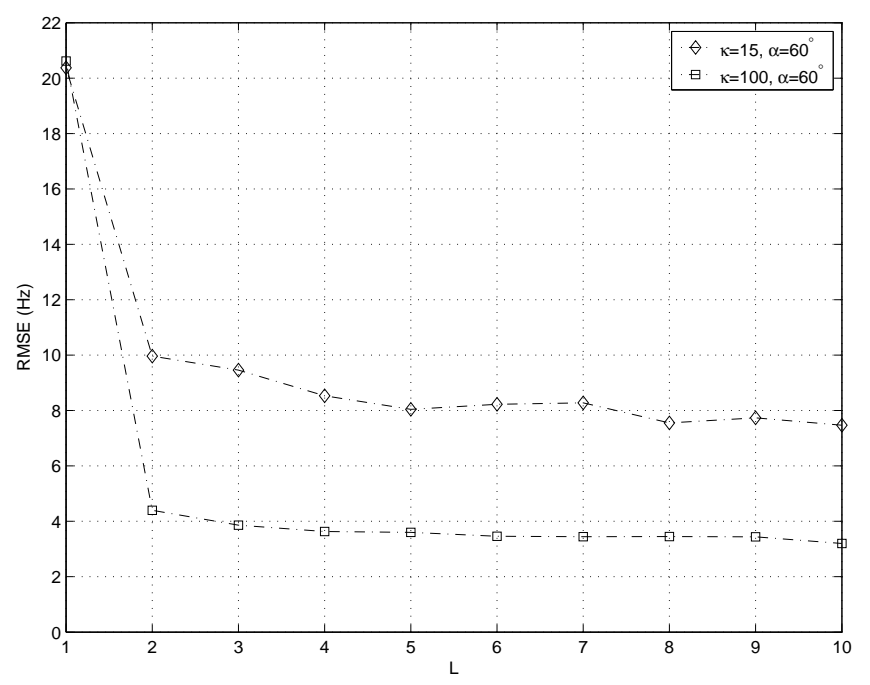

Fig. 9. Effect of the number of antenna elements $L$ on the space-time estimator in a noisy Rayleigh fading channel $\left(\mathrm{SNR}=10 \mathrm{~dB}, f_{D}=41 \mathrm{~Hz}\right.$, $\Delta=0.5)$.

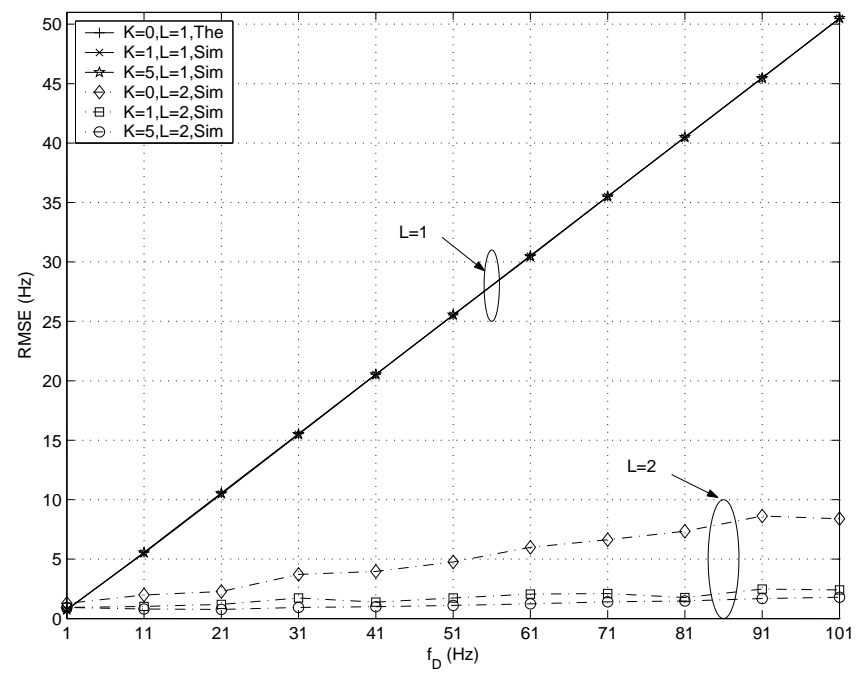

Fig. 10. Performance of the space-time speed estimator ( $\mathrm{SNR}=10 \mathrm{~dB}, L=2$, $\Delta=0.5)$ : Estimation error versus $f_{D}$ for both Rayleigh and Rician multipleantenna channels $\left(\kappa=100, \alpha=60^{\circ}, \alpha_{0}=60^{\circ}\right)$

$\mathrm{Hz}, \kappa=100, \alpha=60^{\circ}$, and $L=2$, in Rayleigh fading, the RMSE changes within $1.5 \mathrm{~Hz}$, as $\Delta$ varies from 0.25 to 1 .

\section{Application to Measured Data}

In this section, we compare our proposed PSD-based estimator in (12) (PSD) with seven other methods: five crossingbased methods which consist of the inphase zero crossing rate estimator (IZCR) [3], envelope level crossing rate estimator (ELCR) [3], inphase rate of maxima estimator (IROM) [3], envelope rate of maxima estimator (EROM) [3], instantaneous frequency zero crossing rate estimator (FZCR) [9], and two covariance-based methods which include the inphase integration estimator (IINT) [3] and envelope integration estimator (EINT) [3]. We also compare our estimator with two nonparametric estimators (32) and (35) which for convenience are denoted as BB and SM respectively. They are all applied

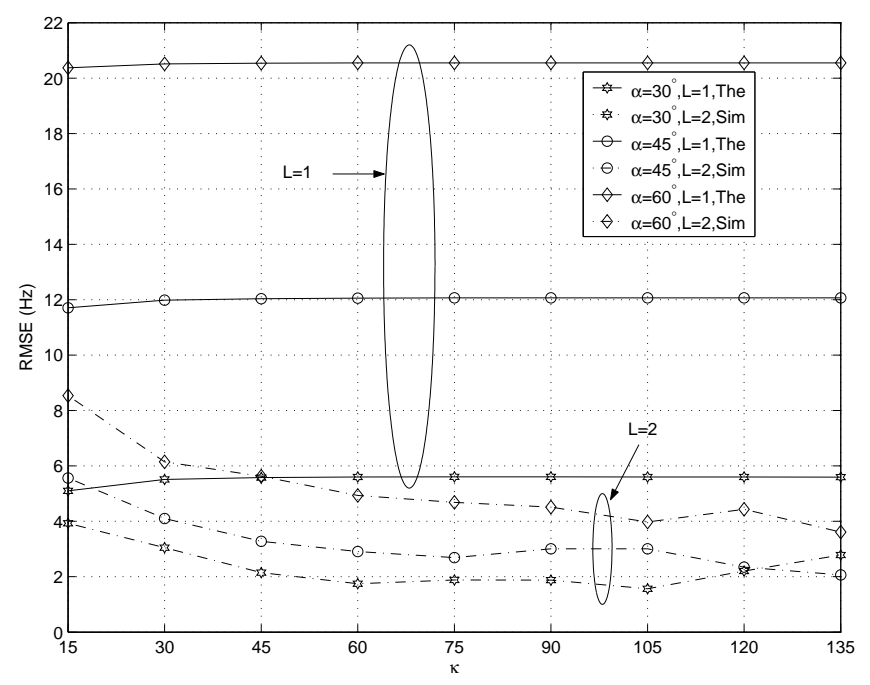

Fig. 11. Performance of the space-time speed estimator ( $\mathrm{SNR}=10 \mathrm{~dB}, L=2$, $\Delta=0.5)$ : Effect of $\kappa$ and $\alpha$ in a Rayleigh multiple-antenna channel $\left(f_{D}=\right.$ $41 \mathrm{~Hz})$

to the narrowband measured data, collected at $910.25 \mathrm{MHz}$, in urban and suburban areas [22]. A vehicle, moving with the constant speed of $15 \mathrm{mph}, f_{D}=20 \mathrm{~Hz}$, has collected sets of narrowband inphase and quadrature components, at different locations, recorded as \#0011, and so on. The signals are filtered by a 42-tap digital Kaiser filter. The bandwidth was $1 \mathrm{kHz}$ to reject out-of-band noise, but large enough to pass through the signal Doppler spectrum unchanged. Each inphase (quadrature) component has approximately 250,000 samples, one sample per 28 microseconds. More information about the data can be found in [22].

Without any further preprocessing and for each record, IZCR, ELCR, IROM, EROM, FZCR, IINT, EINT, BB, SM, and the proposed nonparametric PSD estimator are used to estimate the maximum Doppler frequency from data segments of length $0.56 \mathrm{~ms}, 200$ samples per segment. Each record includes nearly 1250 segments, and for a given method, 1250 estimates are obtained and combined by a simple averaging. Based on Fig. 12, PSD demonstrates the best performance and exhibits more accurate estimates for different records. To see the effect of the number of samples, we chose larger data segments, $1.4 \mathrm{~ms}$ each with 500 samples, and the results show that the performance of FZCR degrades significantly while the RMSE of IZCR, BB and SM decreases, and the PSD is much more consistent and still the best. Overall, our proposed PSD-based speed estimator is able to provide significant performance improvement, when compared with traditional techniques.

\section{CONCLUSION}

In this paper we have proposed a robust speed estimation technique, based on the unique spectral features of the mobile wireless fading channel. As a result, two temporal only speed estimators which are applicable to narrowband and wideband channels, and one space-time speed estimator based on an antenna array, are developed. The effects of noise, nonisotropic 


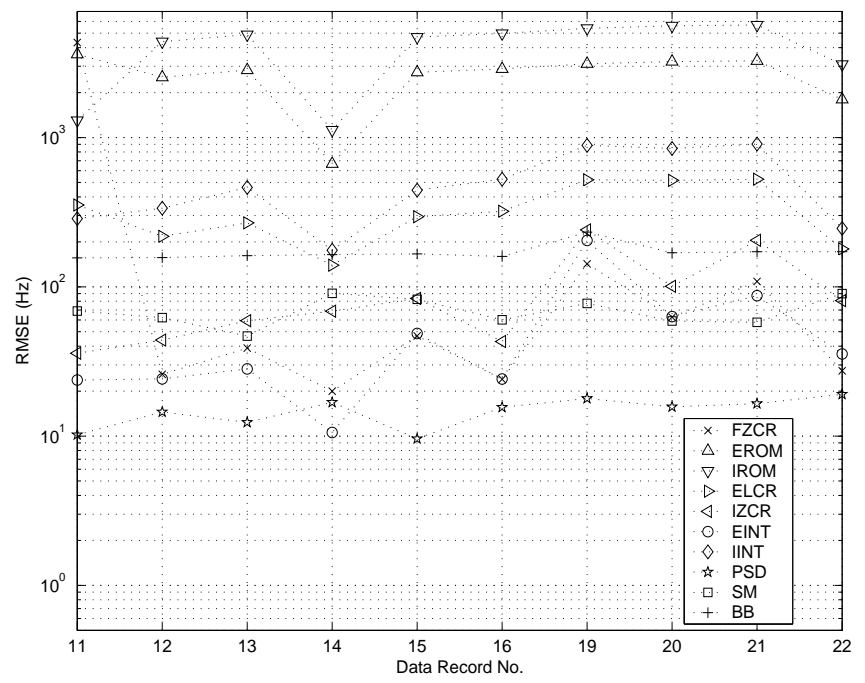

Fig. 12. Performance comparison using the measured data in a narrowband channel, 200 samples per segment.

scattering with two and three dimensional propagations, and line-of-sight are extensively investigated through mathematical performance analysis and Monte Carlo simulations. Comparison with conventional speed estimation methods, using data collected in urban and suburban areas, has demonstrated the utility of the new approach in real-world scenarios.

\section{APPENDIX I}

\section{The PeAk in the Doppler Spectrum}

The power spectral density (PSD) of the unit-power $h^{d}(t)$ for an arbitrary 2-D AOA distribution $p(\phi)$, with a unit-gain isotropic receive antenna can be expressed as [2]

$$
\Omega_{h^{d}}(f)=\frac{1}{\sqrt{f_{D}^{2}-f^{2}}}(p(\phi)+p(-\phi)),
$$

where $\phi=\cos ^{-1}\left(f / f_{D}\right)$. In a heavily nonisotropic scattering, i.e., $\kappa>10, p(\phi)$ is approximately Gaussian with mean $\alpha$ and variance $1 / \kappa$ [27], and we observe $p(\phi) / p(-\phi)=$ $\exp (2 \kappa \phi \alpha)$. Clearly, as $\kappa \rightarrow \infty$ and for a given $f, \Omega_{h^{d}}(f) \approx$ $p(\phi) / \sqrt{f_{D}^{2}-f^{2}}$ when $\alpha$ and $\phi$ have the same sign, and $\Omega_{h^{d}}(f) \approx p(-\phi) / \sqrt{f_{D}^{2}-f^{2}}$, when $\alpha$ and $\phi$ have opposite signs. Without loss of generality, we choose positive $\alpha$ and $\phi$, which yields

$$
\begin{aligned}
\Omega_{h^{d}}(f) \approx & \frac{p(\phi)}{\sqrt{f_{D}^{2}-f^{2}}}=\sqrt{\frac{\kappa}{2 \pi\left(f_{D}^{2}-f^{2}\right)}} \\
& \times \exp \left\{-\frac{\kappa\left(\cos ^{-1}\left(f / f_{D}\right)-\alpha\right)^{2}}{2}\right\} .
\end{aligned}
$$

By taking the derivative of (39) with respect to $f$ and setting it to zero, with the assumption of $f \neq \pm f_{D}$ and some simplifications, we obtain

$$
\frac{1}{\kappa}+\left(\left(\frac{f_{D}}{f}\right)^{2}-1\right)^{\frac{1}{2}}\left(\cos ^{-1}\left(\frac{f}{f_{D}}\right)-\alpha\right)=0 .
$$

When $\kappa \gg 1, f=f_{D} \cos \alpha$ is the root of eq. (40). To verify $f=f_{D} \cos \alpha$ corresponds to the maximum of $\Omega_{h^{d}}(f)$ in (39), we look at the second derivative of (39) with respect to $f$ at $f=f_{D} \cos \alpha$

$$
-\frac{\sqrt{\kappa}(\kappa-4-(\kappa+2) \cos (2 \alpha))}{2 \sqrt{2 \pi} f_{D}^{3} \sin ^{5} \alpha}
$$

which is negative for $\alpha>0$, when $\kappa \gg 1$.

To show that the peak at $f=f_{D} \cos \alpha$ includes most of the unit power of $h^{d}(t)$ as $\kappa \rightarrow \infty$, using the method of Laplace [38], one can obtain the power centered around $f=f_{D} \cos \alpha$, over a sufficiently small bandwidth $\varepsilon$, as follows

$$
\int_{f_{D} \cos \alpha-\varepsilon / 2}^{f_{D} \cos \alpha+\varepsilon / 2} \Omega_{h^{d}}(f) d f \approx \operatorname{erf}(U \sqrt{\kappa / 2})
$$

where $\operatorname{erf}(x)=2 \pi^{-1 / 2} \int_{0}^{x} e^{-t^{2}} d t$ is the error function, and $U=\left|\cos ^{-1}\left(\cos \alpha+0.5 f_{D}^{-1} \varepsilon\right)-\alpha\right|$. Since $U$ is a finite positive number, the above integral converges to 1 as $\kappa \rightarrow \infty$. So, for large $\kappa$, the power is mostly concentrated at $f_{D} \cos \alpha$.

\section{REFERENCES}

[1] M. J. Chu and W. E. Stark, "Effect of mobile velocity on communications in fading channels," IEEE Trans. Veh. Technol., vol. 49, pp. 202-210, Jan. 2000.

[2] G. L. Stüber, Principles of Mobile Communication, 2nd ed. Boston, MA: Kluwer, 2001.

[3] A. Abdi, H. Zhang, and C. Tepedelenlioglu, "A unified approach to performance analysis of speed estimation techniques in mobile communication,” IEEE Trans. Commun., vol. 56, pp. 126-135, Jan. 2008.

[4] J. Holtzman and A. Sampath, "Adaptive averaging methodology for handoffs in cellular systems," IEEE Trans. Veh. Technol., vol. 44, pp. 59-66, Feb. 1995.

[5] K. Anim-Appiah, "On generalized covariance-based velocity estimation,” IEEE Trans. Veh. Technol., vol. 48, pp. 1546-1557, Sept. 1999.

[6] C. Tepedelenlioglu and G. Giannakis, "On velocity estimation and correlation properties of narrow-band mobile communication channels," IEEE Trans. Veh. Technol., vol. 50, pp. 1039-1052, July 2001.

[7] J. Lin and J. G. Proakis, "A parametric method for Doppler spectrum estimation in mobile radio channels," in Proc. Conf. Inf. Sciences and Systems, Baltimore, MD, 1993, pp. 875-880.

[8] K. E. Baddour and N. C. Beaulieu, "Robust Doppler spread estimation in nonisotropic fading channels," IEEE Trans. Wireless Commun., vol. 4, pp. 2677-2682, Nov. 2005.

[9] H. Zhang and A. Abdi, "Mobile speed estimation using diversity combining in fading channels," in Proc. IEEE Global Telecommun. Conf., Dallas, TX, 2004, pp. 3685-3689.

[10] L. Krasny, H. Arslan, D. Koilpillai, and S. Chennakeshu, "Doppler spread estimation in mobile radio systems," IEEE Commun. Lett., vol. 5 , pp. 197-199, May 2001.

[11] A. Dogandzic and B. Zhang, "Estimating Jakes' Doppler power spectrum parameters using the Whittle approximation," IEEE Trans. Signal Processing, vol. 53, pp. 987-1005, Mar. 2005.

[12] R. Narasimhan and D. C. Cox, "Speed estimation in wireless systems using wavelets," IEEE Trans. Commun., vol. 47, pp. 1357-1364, Sept. 1999.

[13] L. Wang, M. Silventoinen, and Z. Honkasalo, "A new algorithm for estimating mobile speed at the TDMA-based cellular system," in Proc. IEEE Veh. Technol. Conf., Atlanta, GA, 1996, pp. 1145-1149.

[14] K. E. Baddour and N. C. Beaulieu, "Nonparametric Doppler spread estimation for narrowband wireless channels," IEEE Trans. Veh. Technol., submitted.

[15] S. Mohanty, "VEPSD: A novel velocity estimation algorithm for nextgeneration wireless systems," IEEE Trans. Wireless Commun., vol. 4, pp. 2655-2660, Nov. 2005.

[16] G. Park, D. Heo, D. Hong, and C. Kang, "A new maximum Doppler frequency estimation algorithm in frequency domain," in Proc. The 8th Int. Conf. Communication Systems, Washington, DC, 2002, pp. 548-552.

[17] T. Aulin, "A modified model for the fading signal at a mobile radio channel,” IEEE Trans. Veh. Technol., vol. 28, pp. 182-203, Aug. 1979.

[18] J. D. Parsons, The Mobile Radio Propagation Channel, 2nd ed. Chichester, England: Wiley, 2000. 
[19] A. D. Spaulding and D. Middleton, "Optimum reception in an impulsive interference environment - part I: Coherent detection," IEEE Trans. Commun., vol. 25, pp. 910-923, Sept. 1977.

[20] J. Haring and A. J. H. Vinck, "Performance bounds for optimum and suboptimum reception under class-A impulsive noise," IEEE Trans. Commun., vol. 50, pp. 1130-1136, July 2002.

[21] R. Janaswamy, Radiowave Propagation and Smart Antennas for Wireless Communications. Boston, MA: Kluwer, 2001.

[22] A. Abdi, J. A. Barger, and M. Kaveh, "A parametric model for the distribution of the angle of arrival and the associated correlation function and power spectrum at the mobile station," IEEE Trans. Veh. Technol., vol. 51, pp. 425-434, May 2002.

[23] R. H. Clarke and W. Khoo, "3-D mobile radio channel statistics," IEEE Trans. Veh. Technol., pp. 798-799, Aug. 1997.

[24] S. Patel and G. Stuber, "Simulation of rayleigh-faded mobile-to-mobile communication channels," IEEE Trans. Commun., pp. 1876-1884, Nov. 2005.

[25] P. Stoica and R. L. Moses, Introduction to Spectral Analysis. Upper Saddle River, New Jersey: Prentice Hall, 1997.

[26] X. Zhao, J. Kivinen, P. Vainikainen, and K. Skog, "Characterization of Doppler spectra for mobile communications at $5.3 \mathrm{GHz}$," IEEE Trans. Veh. Technol., vol. 52, pp. 14-23, Jan. 2003

[27] A. Abdi and M. Kaveh, "Parametric modeling and estimation of the spatial characteristics of a source with local scattering," in Proc. IEEE Int. Conf. Acoust., Speech, Signal Processing, Orlando, FL, 2002, pp. 2821-2824.

[28] W. C. Jakes, Microwave Mobile Communications. New York: Wiley, 1974, ch. Multipath interference, pp. 11-78.

[29] W. C. Y. Lee, "Effects on correlation between two mobile radio basestation antennas," IEEE Trans. Commun., vol. 21, pp. 1214-1224, 1973.

[30] K. I. Pedersen, P. E. Mogensen, and B. H. Fleury, "A stochastic model of the temporal and azimuthal dispersion seen at the base station in outdoor propagation environments," IEEE Trans. Veh. Technol., vol. 49, pp. 437-447, Mar. 2000.

[31] P. Pajusco, "Experimental characterization of D.O.A at the base station in rural and urban area," in Proc. IEEE Veh. Technol. Conf., Ottawa, ON, Canada, 1998, pp. 993-997.

[32] A. Abdi and M. Kaveh, "A versatile spatio-temporal correlation function for mobile fading channels with non-isotropic scattering," in Proc. IEEE Workshop Statistical Signal Array Processing, Pocono Manor, PA, 2000, pp. 58-62.

[33] P. J. Brockwell and R. Davis, Introduction to time series and forecasting, 2nd ed. New York: Springer-Verlag, 2002.

[34] F. D. Neeser and J. L. Massey, "Proper complex random processes with applications to information theory," IEEE Trans. Inform. Theory, vol. 39, pp. 1293-1302, July 1993.

[35] K. Acolatse and A. Abdi, "Efficient simulation of space-time correlated MIMO mobile fading channels," in Proc. IEEE Veh. Technol. Conf., Orlando, FL, 2003, pp. 652-656.

[36] S. Miyamoto, M. Katayama, and N. Morinaga, "Performance analysis of QAM systems under class A impulsive noise environment," IEEE Trans. Electromagn. Compat., vol. 37, pp. 260-267, May 1995.

[37] J. G. Proakis, Digital Communications, 4th ed. New York: McGrawHill, 2001.

[38] E. T. Copson, Asymptotic Expansions. Cambridge, U.K.: Cambridge Univ. Press, 1965

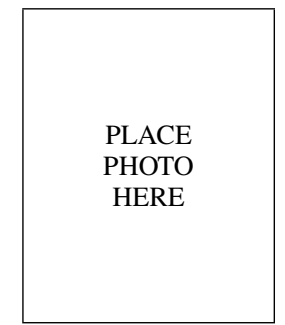

Hong Zhang (S'04-M'07) received the B.S. and M.S. degrees from the Department of Electronic Engineering, Beijing University of Aeronautics and Astronautics (BUAA), Beijing, China, in 1994 and 1997, and the Ph.D. degree from the Department of Electrical and Computer Engineering, New Jersey Institute of Technology (NJIT), Newark, New Jersey in 2007, respectively. From 1997-1999, he was with China Integrated-circuit Design Center (CIDC), Beijing, China. In 1999, he was with Wireless R\&D, Huawei Technologies, Beijing, China. From 19992001, he was with PCS R\&D, Motorola, Beijing, China. From 2002-2006, he was a Research Assistant at the Center for Wireless Communications and Signal Processing Research (CWCSPR) at the Department of Electrical and Computer Engineering, NJIT, Newark, New Jersey. Since 2006, He has been with InterDigital, Inc, King of Prussia, PA. His research interests include wireless communication theory and its applications to current- and futuregeneration mobile communication systems.

Dr. Zhang holds 4 U.S. patents (pending) and is the winner of 2008 New Jersey Inventors Hall of Fame (NJHoF) Graduate Student Award. He is also the recipient of NJIT Hashimoto Fellowship.

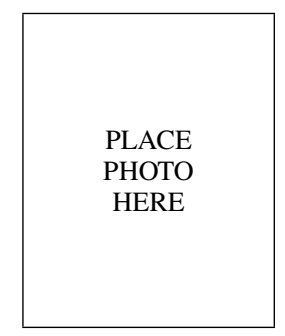

Ali Abdi (S'98, M'01, SM'06) received the Ph.D. degree in electrical engineering from the University of Minnesota, Minneapolis, in 2001. He joined the Department of Electrical and Computer Engineering of New Jersey Institute of Technology (NJIT), Newark, in 2001, where he is currently an Associate Professor. His current research interests include characterization and estimation of wireless channels, digital communication in underwater and terrestrial channels, blind modulation recognition, systems biology and molecular networks. Dr. Abdi was an Associate Editor for IEEE Transactions on Vehicular Technology from 2002 to 2007. He was also the co-chair of the Communication and Information Theory Track of the 2008 IEEE ICCCN (International Conference on Computer Communications and Networks). Dr. Abdi has received 2006 NJIT Excellence in Teaching Award, in the category of Excellence in Team, Interdepartmental, Multidisciplinary, or Non-Traditional Teaching. He has also received 2008 New Jersey Inventors Hall of Fame (NJIHoF) Innovators Award on Acoustic Communication, for his work on underwater acoustic communication. 